Conclusion: The newly described antibodies against GPCR, GF and GFR are highly correlated. Associations with morbidity- and mortality-determining organ involvement indicate their possible functional relevance and novel pathophysiological mechanisms. As new biomarkers, some of the ab have prognostic value for SSc; for other manifestations, their value should be evaluated in further studies.

REFERENCES:

[1] Cabral-Marques, O., Marques, A., Giil, L.M. et al. GPCR-specific autoantibody signatures are associated with physiological and pathological immune homeostasis. Nat Commun 9, 5224 (2018). https://doi.org/10.1038/ s41467-018-07598-9

[2] Riemekasten G, Philippe A, Näther M, et al. Involvement of functional autoantibodies against vascular receptors in systemic sclerosis Annals of the Rheumatic Diseases 2011;70:530-536.

[3] Weigold, F., Günther, J., Pfeiffenberger, M. et al. Antibodies against chemokine receptors CXCR3 and CXCR4 predict progressive deterioration of lung function in patients with systemic sclerosis. Arthritis Res Ther 20, 52 (2018). https://doi.org/10.1186/s13075-018-1545-8

Disclosure of Interests: Kristina Sterner: None declared, Césaire J. K. Fouodo: None declared, Inke König: None declared, Axel Künstner: None declared, Hauke Busch: None declared, Harald Heidecke Shareholder of: Owner of CellTrend, Anja Schumann: None declared, Antje Müller: None declared, Gabriela Riemekasten: None declared, Susanne Schinke Grant/research support from: UCB sponsors EULAR registration fees

DOI: 10.1136/annrheumdis-2021-eular.1504

\section{OP0245 \\ ANTI-S100A4 MONOCLONAL ANTIBODY TREATMENT AMELIORATES SKIN FIBROSIS IN INFLAMMATORY AND NON-INFLAMMATORY PRE-CLINICAL MODELS OF SYSTEMIC SCLEROSIS}

M. Tomčík ${ }^{1}$, T. Trinh-Minh ${ }^{2}$, C. T. Manh² ${ }^{2}$ H. Štorkánová ${ }^{1}$, L. Štorkánová ${ }^{1}$, L. Šenolt ${ }^{1}$, J. Klingelhöfer ${ }^{3}$, R. I. Hussain ${ }^{3}$, J. Hallén ${ }^{3}$, J. H. W. Distler ${ }^{2}{ }^{1}$ Institute of Rheumatology and First Faculty of Medicine, Charles University, Department of Rheumatology, Prague, Czech Republic; ${ }^{2}$ Department of Internal Medicine III and Institute for Clinical Immunology, University of Erlangen-Nuremberg, Erlangen, Department of Internal Medicine III, Erlangen, Germany; ${ }^{3}$ Arxx Therapeutics, Arxx Therapeutics, Oslo, Norway

Background: AX-202 is a monoclonal antibody that inhibits the bioactivity of S100A4. S100A4 is an alarm signal that is released from cells in response to stress or injury and functions as an amplifying mechanism of inflammation and fibrosis in the diseased tissue microenvironment. Previous in vitro studies have found that S100A4 induces fibroblast activation, sensitizes fibroblasts to the effects of TGF $\beta$, drives epithelial-mesenchymal transition, and stimulates monocyte cytokine release (1-3). Moreover, S100A4-/- mice are protected from fibrosis in several animal models (1). In patients with systemic sclerosis (SSc), S100A4 is elevated both in lesional tissue and systemically and correlates with skin involvement, disease activity, and pulmonary function.

Objectives: The aim of this study was to assess the antifibrotic effects of murine AX-202 in two pre-clinical models of SSs reflecting both inflammation-mediated and inflammation non-mediated fibrosis and confirm the in vivo activity of humanized AX-202.

Methods: We first evaluated the effects of murine AX-202 in the bleomycin-induced skin fibrosis model and the tight-skin 1 (Tsk-1) model. In the bleomycin (BLM) model, fibrosis was induced by 3 weeks of BLM s.c. injections followed by 3 weeks of $A X-202$ treatment in parallel with continued BLM s.c. injections. The control groups included $\mathrm{NaCl}$ s.c. injections for 6 weeks, BLM s.c. injections for 6 weeks, or BLM s.c. injections for 3 weeks, followed by $\mathrm{NaCl}$ s.c. injections for 3 weeks. Three dosing regimens of AX-202 were tested: $3.75,7.5$, or $12.5 \mathrm{mg} / \mathrm{kg}$ i.p. every $3^{\text {rd }}$ day. In the Tsk- 1 model, treatment with $7.5 \mathrm{mg} / \mathrm{kg}$ i.p. every $3^{\text {rd }}$ day was administered from week 5 until week 10. The control groups included pa mice, Tsk-1 mice, and Tsk-1 mice treated i.p. with isotype IgG. We subsequently evaluated the effects of humanized AX-202 in the model of BLM-induced skin fibrosis in a similar design as used for the murine AX-202 study. Three dosing regimens were tested: $8 \mathrm{mg} / \mathrm{kg}$ and $16 \mathrm{mg} / \mathrm{kg}$ i.p. every $3^{\text {rd }}$ day and $24 \mathrm{mg} / \mathrm{kg}$ i.v. once weekly.

Results: In the BLM model, murine AX-202 $(7.5 \mathrm{mg} / \mathrm{kg})$ was effective both in the prevention of progression of pre-established skin fibrosis and in the induction of regression of fibrosis as assessed by the dermal thickness $(-55 \%, p<0.0001$ vs BLM for 6 weeks, and $-23 \%, p<0.0001$ vs BLM for 3 weeks), myofibroblast count and hydroxyproline content. Murine AX-202 also ameliorated fibrosis in the Tsk-1 model as assessed by the hypodermal thickness $(-24 \%, p=0.01$ vs Tsk-1 isotype control), myofibroblast count, and hydroxyproline content. In both models, the antifibrotic effects were associated with a reduction in pSMAD3 expression. Humanized AX-202 was effective in the prevention of progression of pre-established skin fibrosis in all doses tested across all endpoints (dermal thickness, myofibroblast counts, hydroxyproline content). In the two groups treated with
$16 \mathrm{mg} / \mathrm{kg}$ i.p. and $24 \mathrm{mg} / \mathrm{kg}$ i.v., humanized AX-202 also induced regression of fibrosis $(-83 \%, p<0.001$, and $-61 \%, p<0.001$ vs BLM for 3 weeks, respectively). Both murine and humanized AX-202 were well tolerated in all study groups in both models.

Conclusion: We demonstrate that AX-202 confers potent antifibrotic effects in complementary models of SSc. These results confirm and expand previous data showing that inhibition of S100A4 by AX-202 is a promising potential therapeutic candidate for disease modification in SSc or other fibrotic conditions.

\section{REFERENCES:}

[1] Tomcik M et al. S100A4 amplifies TGF-beta-induced fibroblast activation in systemic sclerosis. Ann Rheum Dis. 2015;74(9):1748-55.

[2] Cerezo LA et al. The metastasis-associated protein S100A4 promotes the inflammatory response of mononuclear cells via the TLR4 signalling pathway in rheumatoid arthritis. Rheumatology (Oxford). 2014;53(8):1520-6

[3] Fei F, et al. Role of metastasis-induced protein S100A4 in human non-tumor pathophysiologies. Cell Biosci. 2017;7:64

Acknowledgements: The study was supported by Arxx Therapeutics and MHCR 023728.

Disclosure of Interests: Michal Tomčík: None declared, Thuong Trinh-Minh None declared, Cuong Tran Manh: None declared, Hana Štorkánová: None declared, Lenka Štorkánová: None declared, Ladislav Šenolt: None declared Jörg Klingelhöfer Employee of: Arxx Therapeutics, Rizwan I Hussain Employee of: Arxx Therapeutics, Jonas Hallén Employee of: Arxx Therapeutics, Jörg H.W. Distler Shareholder of: the stock owner of 4D Science, Consultant of: Actelion, Active Biotech, Anamar, ARXX, Bayer Pharma, Boehringer Ingelheim, Celgene Galapagos, GSK, Inventiva, JB Therapeutics, Medac, Pfizer, RuiYi and UCB, Grant/research support from: Anamar, Active Biotech, Array Biopharma, ARXX aTyr, BMS, Bayer Pharma, Boehringer Ingelheim, Celgene, Galapagos, GSK Inventiva, Novartis, Sanofi-Aventis, RedX, UCB DOI: 10.1136/annrheumdis-2021-eular.1968

\section{\begin{tabular}{|l|l|l|l|}
\hline OP0246 PREDICTIVE VALUE OF LABORATORY AND & PATY \\
\hline
\end{tabular} INSTRUMENTAL FINDINGS IN THE VERY EARLY DIAGNOSIS OF SYSTEMIC SCLEROSIS. ROLE FOR CXCL4 CHEMOKINE}

M. Cadar $^{1}$, C. Angelelli ${ }^{1}$, G. Pellegrino ${ }^{1}$, K. Stefanantoni ${ }^{1}$, R. Palazzo ${ }^{2}$, R. Lande ${ }^{2}$, L. Frasca ${ }^{2}$, V. Riccieri'. ' Sapienza University of Rome, Dipartimento di Scienze Cliniche Internistiche, Anestesiologiche e Cardiovascolari, Roma, Italy; ${ }^{2}$ Italian National Institute of Health, National Centre for Pre-Clinical and Clinical Drug Research and Evaluation, Pharmacological Research and Experimental Therapy Unit, Roma, Italy

Background: Systemic sclerosis (SSc) is a rare and progressive autoimmune disease, whose diagnosis is difficult in the early stages because of the lack of specific signs and symptoms. Criteria for a Very Early Diagnosis of SSc (VEDOSS) have been proposed to identify those patients affected by undifferentiated connective tissue disease (UCTD) at risk to develop SSc [1]. For the diagnosis of SSc a strict clinical and laboratory follow up is mandatory [2]. CXCL4 chemokine recently proved to be higher in early SSc [3][4][5].

Objectives: Aim of our study was to evaluate at baseline the main clinical-demographic and laboratory parameters in a group of VEDOSS patients, comparing these features during the follow-up, to detect any difference between progressors (P) and non-progressors (NP) into SSc. Furthermore, we dosed plasma levels of CXCL4.

Methods: We included 27 VEDOSS patients, defined by EUSTAR 2011 Criteria and not fulfilling the 2013 ACR/EULAR classification criteria of SSc, attending the Rheumatology Unit of Policlinico Umberto I in Rome from 2009 to 2020. Demographic, laboratory and instrumental features were analyzed, and, after a mean follow-up of $5.7 \pm 1,7$ years, we compared the P to NP patients. Having obtained written informed consent, blood samples were taken at baseline to measure plasma levels of CXCL4 chemokine using an ELISA assay.

Results: At baseline the 27 VEDOSS patients (mean age $53.2 \pm 13.5$ years, all females) had ANA positivity in $25(93 \%)$ cases and Raynaud's phenomenon in $25(93 \%)$ cases. In a mean follow-up time of $43.5 \pm 23.1$ weeks from the firs clinical examination, 15 (55\%) patients were classified as $\mathrm{P}$ into SSc. These $\mathrm{P}$ patients showed a significant association with SSc specific antibodies such as anti-Centromere, anti-Scl70 and anti-RNAPIII $(p=0.014)$ as well as with a specific "Scleroderma pattern" at the nailfold capillaroscopy $(p=0.022)$ respect to those NP into SSc. A group of 6 P patients evolved in less than 24 month (mean 19,6 6,8 weeks) and were defined "Fast Progressor". They were also significantly associated with those autoantibodies considered as having a worse prognosis such as anti-Scl70 and anti-RNAPIII ( $p=0.005)$, and had a shorter duration of RP (88 vs 189.3 months) and a younger mean age ( 49.5 vs 55.4 years) respect to "Slow Progressor" (SSc evolving in > 24 months). At baseline we detected significantly higher median plasma levels of CXCL4 in the 27 VEDOSS patients compared to 10 healthy subjects $(9024 \pm 10559 \mathrm{pg} / \mathrm{ml}$ versus $348,5 \pm 684,3 \mathrm{pg} / \mathrm{ml} ; \mathrm{p}=0.0047)$. We also noticed a trend for lower CXCL4 levels in the "Fast Progressor" than in 
the "Slow Progressor" (3303 $\pm 6065 \mathrm{pg} / \mathrm{ml}$ vs $13300 \pm 10308 \mathrm{pg} / \mathrm{ml} ; \mathrm{p}=0.052)$ without reaching a significant value, due probably to the low number of cases. Conclusion: Our study confirms that the presence of specific autoantibodies and capillaroscopic abnormalities correlate to an increased risk of developing SSc in patients with UCTD [6]. Beside we found significantly higher levels of CXCL4 in our 27 VEDOSS patients respect to controls, in agreement with other authors showing the association of this chemokine with early stages and specific organ involvement [4][5][7]. The finding of CXCL4 lower levels in "fast progressor" cases is consistent with our recent report of anti-CXCL4 antibodies in patients with early SSc, determining lower levels of this antigen [7]. We need deeper investigations to better evaluate the role of CXCL4 in the different stages of SSc.

REFERENCES:

[1] J. Avouac et al., Ann. Rheum. Dis., 2011.

[2] F. van den Hoogen et al., Ann. Rheum. Dis., 2013.

[3] G. Valentini et al., Clin. Exp. Med., 2017.

[4] R. Lande et al., Nat. Commun., 2019.

[5] L. van Bon et al., N. Engl. J. Med., 2014.

[6] M. Vasile et al., Clin. Exp. Rheumatol., 2018.

[7] R. Lande et al., Int. J. Mol. Sci., 2020.

Disclosure of Interests: None declared

DOI: 10.1136/annrheumdis-2021-eular.2188

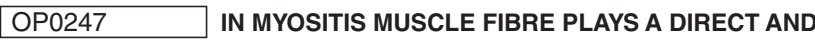 CRITICAL ROLE IN THERAPEUTIC RESPONSE TO GLUCOCORTICOIDS}

M. Giannini ${ }^{1}$, G. Laverny ${ }^{2}$, D. Duteil ${ }^{2}$, D. Rovito ${ }^{2}$, A. L. Charles ${ }^{3}$, B. Geny ${ }^{4}$, D. Metzger ${ }^{2}$, A. Meyer ${ }^{5} .{ }^{1}$ University of Strasbourg, Service de Physiologie et d'explorations fonctionnelles, Nouvel Hopital Civil, Centre de Référence des Maladies Autoimmunes Rares, Fédération de Médecine Translationnelle, UR3072, Strasbourg, Strasbourg, France; ${ }^{2}$ University of Strasbourg, CNRS UMR7104, INSERM U1258, IGBMC, F-67400 Illkirch, Illkirch, France; ${ }^{3}$ University of Strasbourg, Fédération de Médecine translationnelle, UR3072, Strasbourg, Strasbourg, France; ${ }^{4}$ University of Strasbourg, Service de Physiologie et d'explorations fonctionnelles, Nouvel Hopital Civil, Fédération de Médecine Translationnelle, UR3072, Strasbourg, Strasbourg, France; ${ }^{5}$ University of Strasbourg, Service de Physiologie et d'explorations fonctionnelles, Nouvel Hopital Civil, Centre de Référence des Maladies Autoimmunes Rares, Fédération de Médecine Translationnelle, UR3072, Strasbourg, Strasbourg, France

Background: Myositis are rare autoimmune diseases, affecting more women than men, characterized by chronic inflammation of skeletal muscle causing muscle weakness, decreased quality of life and increased mortality.

Glucocorticoids $(\mathrm{GC})$ are potent anti-inflammatory drugs, and are the first line treatment of myositis. They improve muscle strength of myositis patients (therapeutic effect), yet muscle recovery is generally only partial. Moreover, GC have an iatrogenic effect on skeletal muscle fibre leading to steroid myopathy. Thus myositis care has to be improved. Despite the autoimmune terrain of myositis, our team has recently shown that muscle fibres themselves develop immuno-metabolic modifications that participate to muscle weakness and perpetuation of the disease ${ }^{1}$. GC effects are mediated by the glucocorticoid receptor (GR), which is expressed in various cell types including immune cells and myofibres, but the cells mediating therapeutic responses remain to be determined.

Objectives: Unravel the mechanisms underlying the therapeutic effect of GC in myositis, particularly elucidate the role of skeletal muscle fibres.

Methods: Experimental myositis was induced in eight to ten week-old C57BL/6J female mice by a single intradermal injection of part of skeletal muscle fast-type $C$ protein along with Freund's adjuvant and an intraperitoneal (IP) injection of pertussis toxin, as previously described ${ }^{2}$. Prednisone (PDN) was administered 14 days (D) after the immunization at $1 \mathrm{mg} / \mathrm{kg} /$ day for 7 days by gavage. Mice were euthanized 21 days after myositis induction. Muscle strength was assessed by grip test at $\mathrm{D} 0$, before the $1^{\text {st }}$ PDN administration (D 14) and the day before sacrifice (D 20). To investigate whether the PDN effects are mediated by myofibre, we generated transgenic mice carrying two LoxP sites within the GR gene in muscle, expressing the tamoxifen-inducible Cre-ERT2 recombinase selectively in skeletal muscle fibre (HSA-CreERT2/GR L2/L2). Tamoxifen ( $1 \mathrm{mg} /$ day for 5 days by IP injection) was administered 9 days after immunization to induce GR ablation selectively in skeletal muscle fibres (GR(i)skm-/- mice). Similar treatments were applied to GR L2/L2 that do not express Cre-ER(T2), and served as controls.

We compared 4 groups of myositis mice, GR L2/L2 treated by PDN ( $n=9$ ) or vehicle $(n=9)$ and $G R(i) \mathrm{skm}^{-/}$treated by PDN $(n=10)$ or vehicle $(n=10)$, by grip test and at the histological level (hematoxylin-eosin (HE) and Gomori trichrome (GT) staining). Moreover, LC3 expression was studied by RTqPCR and western blot.
Results: Muscle strength was decreased in both GR L2/L2 and GR(i)skmmyositis mice from D 14 to D 20. GR L2/L2 myositis mice recovered muscle strength after PDN treatment; no significant difference compared to $D 0$ was detected. In contrast, PDN did not improve muscle strength in GR(i)skm ${ }^{-/}$myositis mice (Figure 1).

HE and GT staining did not reveal quantitative differences in inflammatory infiltrate. Necrotic and degenerative fibres were detected in the 4 groups. At RTqPCR, LC3, an autophagy marker, was upregulated in PDN-treated GR L2/ L2 myositis mice compared to untreated GR L2/L2 myositis mice; moreover it was 2-fold more expressed in PDN-treated GR L2/L2 myositis mice compared to PDN-treated $\mathrm{GR}(\mathrm{i}) \mathrm{skm}^{-/}$mice.

Conclusion: GR in skeletal muscle fibre is crucial to mediate the therapeutic response to GC in a murine model of myositis. Autophagy is one of the candidate pathways controlled by myofibre GR underlying this effect.

\section{REFERENCES:}

[1] Meyer A et al. IFN- $\beta$-induced reactive oxygen species and mitochondrial damage contribute to muscle impairment and inflammation maintenance in

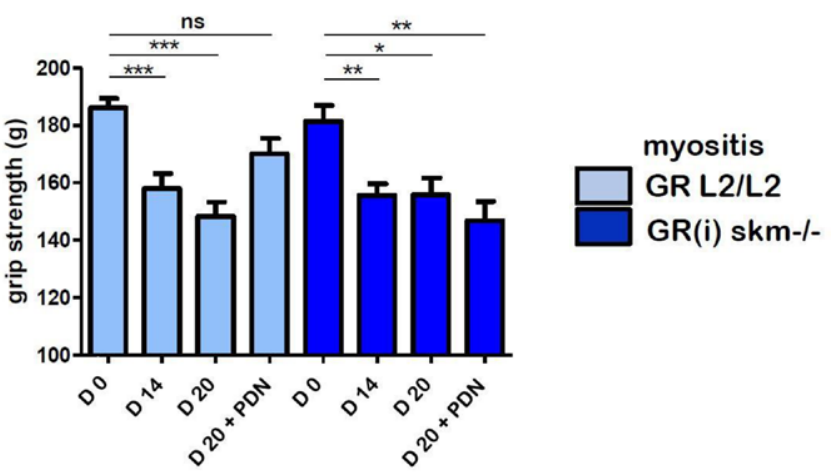

Figure 1. Grip test analysis in GR L2/L2 and GR(i) skm-1myositis mice. ns, not significant; ${ }^{\star} p<0.01 ;{ }^{\star \star} p<0.001$ *** $p<0.0001$

dermatomyositis. Acta Neuropathol. 2017 Oct;134(4):655-666.

[2] Sugihara T et al. A new murine model to define the critical pathologic and therapeutic mediators of polymyositis. Arthritis Rheum. 2007 Apr;56(4):1304-14 Disclosure of Interests: None declared DOI: 10.1136/annrheumdis-2021-eular.2863

\section{FOR FIBROTIC DISEASES}

E. Pachera ${ }^{1}$, G. Kania ${ }^{1}$, A. Juengel ${ }^{1,2}$, M. Calcagni ${ }^{3}$, O. Distler ${ }^{1,2} \cdot{ }^{1}$ Department $^{2}$ of Rheumatology, Center of Experimental Rheumatology, University Hospital Zurich, University of Zurich, Zurich, Switzerland; ${ }^{2}$ Department of Physical Medicine and Rheumatology, Balgrist University Hospital, University of Zurich, Zurich, Switzerland; ${ }^{3}$ Department of Hand Surgery, Balgrist University Hospital, University of Zurich, Zurich, Switzerland

Background: Traditional preclinical approaches, such as two-dimensional cell culture and animal models, are often inadequate to mimic the pathophysiologica features of complex diseases such as systemic sclerosis (SSc). Human specific targets, such as the recently described pro-fibrotic long non coding RNA

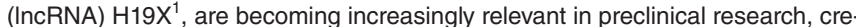
ating the need of new strategies and tools in translational medicine. The employment of novel three-dimensional (3D) culture systems, where multiple cell types are included, is filling an important gap left by the traditional preclinical methods. Objectives: To develop an easy to produce 3D fibrotic skin microtissues mode for translational proof of concept studies.

Methods: Two thousand five hundred dermal fibroblasts isolated from skin of SSc patients were seeded in ultra-low attachment 96-well plates. Fibroblast were let to aggregate into spheres for $48 \mathrm{~h}$. Two thousand five hundred primary norma human keratinocytes were added to the culture and let to layer onto the fibroblast spheres for $72 \mathrm{~h}$. H19X silencing experiments were used as proof of concept studies. H19X silencing with antisense oligonucleotides or transfections with a scrambled control were performed in fibroblasts prior to the sphere formation fo $24 \mathrm{~h}$. TGF $\beta(10 \mathrm{ng} / \mathrm{ml})$ was added to microtissue to exacerbate the fibrotic phenotype. Haematoxylin eosin staining as well as immunohistochemistry staining for vimentin and cytokeratin 10 was performed. Skin microtissues were processed for RNA and protein isolation. Pro-collagen l $\alpha 1$ and fibronectin were quantified in the supernatants with ELISA. 